ENCYCLOPEDDIE Encyclopédie berbère

BERBERE

$26 \mid 2004$

26 | Judaïsme - Kabylie

\title{
Kabylie : Dialectologie
}

K. Naït-Zerrad

\section{OpenEdition}

Journals

Édition électronique

URL : http://journals.openedition.org/encyclopedieberbere/1433

DOI : 10.4000/encyclopedieberbere.1433

ISSN : 2262-7197

\section{Éditeur}

Peeters Publishers

\section{Édition imprimée}

Date de publication : 1 mai 2004

Pagination : 4067-4070

ISBN : 2-7449-0452-X

ISSN : 1015-7344

\section{Référence électronique}

K. Naït-Zerrad, « Kabylie : Dialectologie », Encyclopédie berbère [En ligne], 26 | 2004, document K16, mis en ligne le 01 juin 2011, consulté le 15 décembre 2020. URL : http://journals.openedition.org/ encyclopedieberbere/1433; DOI : https://doi.org/10.4000/encyclopedieberbere.1433

Ce document a été généré automatiquement le 15 décembre 2020.

(c) Tous droits réservés 


\section{Kabylie : Dialectologie}

\section{K. Naït-Zerrad}

1 Les berbérisants regroupent les parlers berbères en variétés régionales ou aires dialectales - comme le chleuh, le kabyle, le chaoui, le touareg, le rifain, etc. - à l'intérieur desquelles l'intercompréhension est normalement assurée. Ces grands ensembles sont eux-mêmes constitués de sous-variétés - représentées chacune par une confédération, une tribu ou un groupe de villages - possédant des particularités spécifiques. Celles-ci ne sont pas seulement restreintes au vocabulaire mais touchent également la phonétique et la morphosyntaxe. (Sur la distinction langue/dialecte/ parler, voir Chaker, EBXV, 1995).

2 Pour des raisons historiques, les études berbères sont dominées depuis le début du $\mathrm{xx}^{\mathrm{e}}$ siècle par des travaux portant sur la Kabylie occidentale (Grande Kabylie) aussi bien à l'université (André Basset, Boulifa...) que dans les publications du Fichier de Documentation berbère fondé par les Pères Blancs en 1946 à Fort-National (Larbaa Nat Iraten). Signalons ici les Etudes de géographie linguistique en Kabylie (1929) d'André Basset, un des rares travaux couvrant la totalité de la Kabylie et portant « sur quelques termes berbères concernant le corps humain ».

3 Après l'indépendance, et jusqu'à une période récente, cette situation perdure, que ce soit en Kabylie ou en émigration, la majorité des acteurs (universitaires ou militants associatifs) étant originaires de la Kabylie occidentale (M. Mammeri, S. Chaker...).

4 Le reste de la Kabylie a donc été le parent pauvre des études berbères, on peut citer néanmoins (outre des notes grammaticales et des textes dans Hanoteau, Basset, Bensedira...) :

- pour sa partie orientale et extrême orientale (Petite Kabylie), le Dictionnaire françaiskabyle (parlers de Bejaia et de ses environs) publié en 1844 par lequel les études berbères ont, paradoxalement, été inaugurées ; les textes de Leblanc de Prebois (1897) et les «Contes merveilleux et fables » $(\mathrm{FDB}, 1976)$ pour les Ait-Abbas ; Slimane Rahmani $(1933,1939)$ et Genevois (1955) pour les parlers de la Kabylie maritime et ses environs (Aoqas, Ait-Mbarek)

- pour sa partie extrême occidentale (Draa El Mizan, Boghni, Tizi-Ghennif...), nous 
n'avons pratiquement aucun texte ni aucune étude, si ce n'est un fascicule consacré aux potières de la région de Bu-Nuh, publié par le FDB (Louis de Vincennes, 1971).

Ce vide commence cependant à être comblé à la faveur des travaux de chercheurs originaires de Kabylie orientale (Allaoua 1988, 1994 ; Rabhi 1995 ; Madoui 1995...) en particulier avec l'ouverture du département de langue et culture amazighes de l'université de Béjaia. Ajoutons que l'on a signalé dans Naït-Zerrad 1994, 1995 et 2001a les particularités phonétiques, morphosyntaxiques et lexicales des parlers orientaux et occidentaux.

La situation linguistique kabyle est beaucoup plus complexe qu'une simple distinction entre Grande Kabylie et Petite Kabylie. Ces dénominations géographiques ont une pertinence linguistique limitée, d'autant que l'on ne sait pas toujours ce que cela recouvre exactement sous la plume des auteurs. Les parlers kabyles constituent un continuum dialectal et il est difficile d'établir une classification à partir d'un faisceau d'isoglosses, les données étant souvent enchevetrées. Par exemple, Basset (1929) montre une variation importante pour "mollet» alors qu'elle est beaucoup plus restreinte pour «oreille». Les sous-variétés conti-guës sont linguistiquement très proches alors que celles situées aux extrêmes sont plus proches du dialecte avec lequel elles sont en contact (le chaoui pour la Kabylie extrême orientale) et l'intercompréhension entre elles est plus difficile.

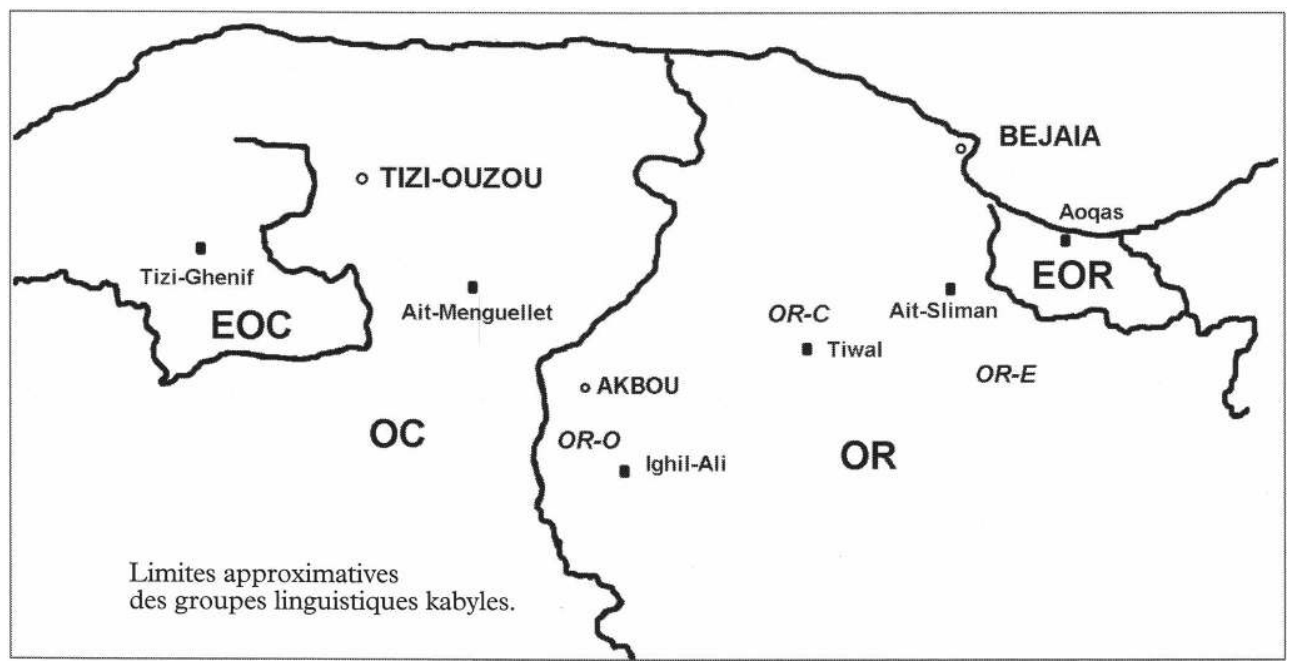

7 On peut néanmoins envisager au moins quatre groupes linguistiques plus ou moins homogènes :

- extrême occidental (EOC : Tizi-Ghennif, Boghni, Draa el Mizan...),

-occidental (OC : At Menguellat, At Yiraten, At Aïssi, At Yanni...),

- oriental (OR-Ouest: At Mlikeche, At Abbas, OR-Centre : At Aïdel, At Khiar, OR-Est : At Sliman...)

-extrême oriental (EOR : Aoqas, Melbou, At Smail...),

que l'on peut encore subdiviser et affiner... Cette distribution se fonde sur plusieurs critères phonétiques, morphosyntaxiques et lexicaux. Nous en proposons quelques-uns dans les lignes qui suivent. À l'intérieur d'un groupe, il peut bien entendu exister des différences sur tel ou tel point très particulier dans tel ou tel village... On ne notera ici que les tendances lourdes (Cf. également Naït-Zerrad 2001b) :

EOC : Tizi-Ghennif (notes personnelles)

OC : Ait-Menguellat (Dallet 1982) 
OR-Ouest : Ighil-Ali (notes personnelles), OR-Centre : Tiwal (notes personnelles), OR-Est : Ait-Slimane (Allaoua 1988)

EOR : Aoqas (Rabhi 1995), Madoui 1995 et notes personnelles

\section{Phonétique : réalisations différentes selon les groupes}

Absence de certaines particules préverbales en OR et EOR $(l a, . .$.

- L'usage courant de la particule $(t) t u \gamma$ comme auxiliaire pour le passé en EOC et EOR (v. également Naït-Zerrad 1995, 2000 et Chaker 1997).

- La grammaticalisation du verbe ini «dire» en OR-Ouest (At Abbas) qui s'emploie comme conjonction.

\section{3. système verbal}

14 - la désinence personnelle de l'impératif masculin pluriel chez les At Abbas se présente sous la forme ----wt quand le thème verbal se termine par une consonne, en regard de la forme sans semi-voyelle ----t attestée dans le reste du domaine kabyle.

- les verbes de qualité : Les At Slimane ont une « conjugaison " particulière qui consiste à employer les affixes personnels directs après le thème verbal de prétérit pour indiquer la personne. Dans les autres parlers, on utilise un paradigme spécifique d'indices personnels suffixés à ce thème. En Kabylie EOR, cette conjugaison semble avoir disparue.

- le préverbe de l'aoriste $a d / a(\mathrm{EOC}, \mathrm{OC}, \mathrm{OR}) \sim d i / i(\mathrm{EOR})$

- les particules d'orientation $d$ et $n$ : cette dernière n'est pas attestée en Kabylie orientale et extrême orientale. 
- la conjugaison des verbes forts du type $\mathrm{CCu}$ et $\mathrm{CC}$ avec respectivement l'apophonie $\mathrm{u} /$ (i/a) et $\varnothing /(i / a)[i$ pour les personnes 1 et 2 du singulier et $a$ pour le reste] en EOC, OC, OR-Ouest et $\mathrm{u} /(\mathrm{i} / \mathrm{a} / \mathrm{i} / \mathrm{a})$ et $\varnothing /(\mathrm{i} / \mathrm{a} / \mathrm{i} / \mathrm{a})$ [i pour les $\mathrm{p} .1 \mathrm{du}$ singulier et 2 singulier et pluriel, $a$ pour le reste] en OR-Centre et Est.

Tableau des variations phonétiques et morphosyntaxiques

\begin{tabular}{|c|c|c|c|c|c|c|}
\hline EOC & $\begin{array}{l}\text { OC } \\
\text { Tizi-Ghennif }\end{array}$ & $\begin{array}{l}\text { OR-Ouest } \\
\text { Ait Manguellat }\end{array}$ & $\begin{array}{l}\text { OR-Centre } \\
\text { At Abbas }\end{array}$ & $\begin{array}{l}\text { OR-Est } \\
\text { At Aidel, At Khiar }\end{array}$ & $\begin{array}{l}\text { EOR } \\
\text { At Sliman }\end{array}$ & Aoqas \\
\hline Préverbe & $A d \mid a$ & $a d / a$ & $a d / a$ & $a d / a$ & $a d / a$ & $d i / i$ \\
\hline$t t \mid t s$ & $t t s$ & tt & $t t$ & $t t$ & $t t / t t$ & $t t$ \\
\hline$D / n$ & $N$ & $n$ & - & - & - & - \\
\hline labial. & + & + & + & + & $-(+)$ & $-(+)$ \\
\hline$(t) t u \gamma$ & tuj & - & - & - & $(t t u \gamma)$ & $t t u \gamma$ \\
\hline Qualité & + & + & + & + & + affixes & - \\
\hline$N+w$ & $B b^{(w)}$ & $b b^{(w)}$ & $g g^{(w)}$ & $w w$ & $w w$ & $w w$ \\
\hline$N+y$ & $G g$ & $g g$ & $G g$ & $y y$ & $y y$ & $y y$ \\
\hline Impératif & $--t$ & $-t$ & $--v t$ & $--t$ & $--t$ & $--t$ \\
\hline Apophon. & ila & $i / a$ & ila & ilalila & ilalila & $i \mid a$ \\
\hline$d$ & $d$ & $d$ & $d$ & $t$ & $t$ & $t$ \\
\hline
\end{tabular}

\section{Lexique}

Pour ce qui est du vocabulaire, on a déjà évoqué l'ouvrage de Basset (1929) qui montre bien - sur les 20 entrées portant sur le corps humain - que l'on ne peut définir de frontière nette entre les 4 groupes EOC, OC, OR et EOR.

Les exemples du tableau ci-dessous montrent la variation entre les différents groupes, étant entendu que cet échantillon ne peut être généralisé, le vocabulaire étant pour une large part commun avec une réserve pour la partie la plus orientale du groupe EOR. Certaines unités lexicales ne sont attestées que dans certains groupes: comme kel «passer la journée », iglifez. « ruminat » chez les At Abbas, ikseb « renard » chez les At Abbas et At Aïdel, etc. que l'on retrouve dans d'autres dialectes. Deux remarques à propos du tableau:

- « rêver » : la forme argu a perdu la première radicale $\left(<{ }^{*} H / W R G\right)$ qui s'est maintenue dans bareg et bureg. On comparera avec les autres parlers: Maroc central, chleuh warg, warga ; touareg harğet, Ghadamès berğ...

- « être épais » : la radicale perdue ${ }^{*} H / W$ (vocalisée ou présente sous la forme de la semiconsonne [w] en EOC, OC et OR-Ouest s'est maintenue en OR-Est et EOR: (i)zhur (Cf. touareg : Ahaggar huhər, Adrar des Ifoghas cuhār, Oudalan zuwār)

On observe donc des variations phonétiques, morphologiques, syntaxiques et lexicales circonscrites à des sous-régions particulières qui permettent de dégager des groupes ayant une certaine unité. Il reste cependant que de vraies frontières linguistiques ne peuvent être tracées étant donné que certains éléments (comme tuү) se retrouvent parfois aux extrêmités du domaine. Ces quelques remarques montrent l'intérêt qu'il y aurait à disposer rapidement de monographies pour les parlers représentant les sousvariétés peu ou non encore documentées pour une meilleure et plus fine connaissance 
de la géographie linguistique de la Kabylie et en conséquence les implications que l'on peut en tirer pour la standardisation du kabyle.

Exemples de variations lexicales

\begin{tabular}{|c|c|c|c|c|c|c|}
\hline EOC & $\begin{array}{l}\text { OC } \\
\text { Tizi-Ghennif }\end{array}$ & $\begin{array}{l}\text { OR -Ouest } \\
\text { Ait Manguellat } \\
\end{array}$ & $\begin{array}{l}\text { OR-Centre } \\
\text { At Abbas }\end{array}$ & $\begin{array}{l}\text { OR-Est } \\
\text { At Aïdel, At Khiar }\end{array}$ & $\begin{array}{l}\text { EOR } \\
\text { At Sliman }\end{array}$ & Aoqas \\
\hline Oreille & amezzu & amezzu & amezzuy & amezzuy & imejj & imejj \\
\hline chevelure & rric & acebbublaceččuy & acekkub & acekkub & cceer & cceer/acekkut \\
\hline Acheter & $a \gamma$ & $a \gamma$ & $a \gamma$ & ay & $a \gamma$ & ase $/$ sey \\
\hline Lait & $a k^{w} e f f a y$ & ayefk $(i)$ & ayefki & ayefki & ayefki & ayekfi \\
\hline Rêver & bargu & $\operatorname{argu}$ & $\operatorname{argu}$ & $\operatorname{argu}$ & $\operatorname{argu}$ & bureg \\
\hline Front & anyir & anyir & ayendur & ayendur & ayendur & авеппит \\
\hline Épais & $z u r$ & zur, zwr & $z u r, z w r$ & $z u r, z w r$ & izhur & zhur \\
\hline
\end{tabular}

\section{BIBLIOGRAPHIE}

voir notice $\mathrm{K} 15$

INDEX

Mots-clés : Dialectologie, Kabylie, Linguistique 